\title{
Pharyngeal trauma: Two unique case reports
}

\author{
Candice Colby, Charles Moore
}

Department of Otolaryngology-Head and Neck Surgery, Emory University, Atlanta, USA

Email: ccolby2@emory.edu

Received 23 May 2012; revised 1 July 2012; accepted 29 July 2012

\begin{abstract}
The neck is a complex pathway for numerous critical structures and is defined by layers of fascial planes and spaces. Traumatic interruption of these planes can occur with injury to any of the mucosal structures within the head and neck, and is often seen as a consequence of pharyngeal injury. Here, the identification and management of two interesting and unique cases of penetrating oropharyngeal trauma are discussed: oropharyngeal trauma following abuse, and a self-inflicted pharyngeal wound with subsequent foreign body ingestion. We also review the literature regarding diagnosis, treatment, and management of traumatic pharyngeal perforation, and discuss possible complications.
\end{abstract}

Keywords: Pharynx; Trauma; Penetrating Injury; Neck; Foreign Body

\section{INTRODUCTION}

The neck is a complex pathway for numerous critical structures whose anatomy can be difficult to fully understand. It is defined by multiple spaces with a variety of nomenclature systems that may further cloud the picture. Division of these spaces occurs through multiple layers of cervical fascia, with the initial division between the superficial and deep layers. The superficial layer separates the skin and subcutaneous tissue from the platysma and muscles of facial expression, while the deep layer is further divided into the superficial, middle, and deep layers.

These fascial layers establish planes and spaces within the neck, and separate multiple critical structures into these particular spaces. Interruption of these planes can allow inflammatory, neoplastic, and traumatic processes to spread with devastating consequences. Traumatic interruption of these planes can occur with injury to any of the mucosal structures within the head and neck, and is often seen as a consequence of pharyngeal injury. In this article, we describe two unique cases of penetrating oropharyngeal insult that led to traumatic interruption of the fascial planes of the neck, and review the literature re- garding management of similar traumatic injuries.

\section{CASE REPORT}

A 49-year-old female presented to an urban emergency department following an assault by her partner, with whom she had an abusive history. During a previous episode three years prior to presentation, the patient sustained a mandible fracture that required open reduction and internal fixation. During this particular altercation, the patient suffered multiple blows and kicks to the face and head. Her assailant also inserted his fingers into the patient's mouth during the assault in an attempt to "rip her throat out”.

On initial examination, the patient was clinically stable and in no respiratory distress. She was mildly anxious and complaining of severe odynophagia and neck pain. There was no crepitus upon palpation of the neck and no tracheal deviation noted. Multiple sub-centimeter, well-approximated lacerations were noted in the oropharynx without active bleeding. Her mandibular fixation plates were palpable but not exposed, and her occlusion was intact.

Computed tomography (CT) demonstrated air in the pharyngeal tissues beginning at the level of the oropharynx, extending into the retropharyngeal space, and laterally within the carotid sheath. There was no injury to the esophagus. Air was also noted to be surrounding the great vessels (Figures 1(a)-(b)), and there was disunion of prior fixation site of the mandible. The air collections were judged to be a result of the multiple oropharyngeal lacerations the patient received during the oral cavity trauma.

Broad-spectrum antibiotics and analgesics were administered in the emergency department, and the patient was admitted for observation with nothing by mouth and repeat neurologic examinations. The patient never developed any signs or symptoms of clinical instability, deep space neck infection, or mediastinitis. Following this period of conservative management, the patient passed an oral intake challenge and was discharged home without the need for nasogastric feeding. Management of her mandibular fracture was deferred to the surgeon who performed the initial repair, and she was subsequently lost to follow-up. 


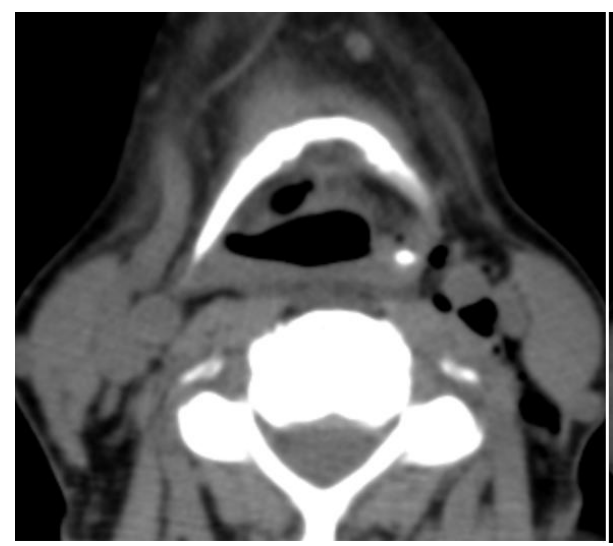

(a)

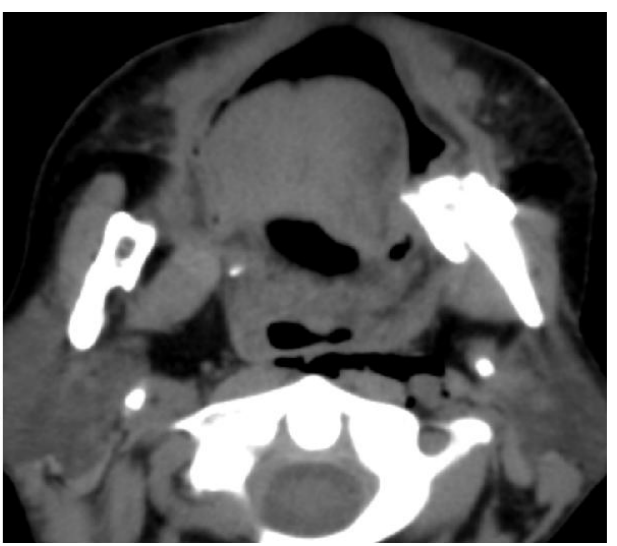

(b)

Figure 1. (a) \& (b): Non-contrast CT of patient with retropharyngeal air tracking to carotid sheath, surrounding the common carotid artery and jugular vein.

\section{SECOND CASE}

A 37-year-old male prison inmate presented to an emergency room following reports of razor blade ingestion as an apparent suicide attempt. The patient had an extensive past medical history that included paranoid schizophrenia, with prior suicide attempts by ingestion of foreign materials. His prior ingestions had required multiple trips to the operating room for exploratory laparotomy with removal of foreign objects including plastic utensils and a toothbrush. His paranoid features made his history difficult to obtain; however due to report of ingestion, a plain film radiograph of the chest and abdomen was performed. No foreign body was identified on these films. Nevertheless, secondary to the patient's extensive history, the gastroenterology service was consulted and performed esphagogastroduodenoscopy to search for retained any foreign body or evidence of trauma. On endoscopy, an approximately two centimeter tear in the posterolateral pharyngeal wall was found (Figure 2), with no distal trauma to the esophagus or stomach, and no foreign body material identified.

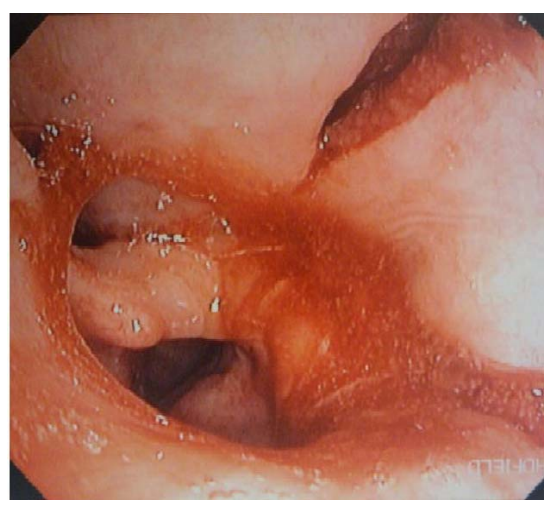

Figure 2. Endoscopic view of self-inflicted posterior pharyngeal wall lacerationthe larynx in shown below the laceration.
The patient began to complain of chest pain while in the trauma bay. His vitals remained normal throughout this time. CT of the neck and chest were performed to further evaluate the pharyngotomy and mediastium, and findings were suspicious for foreign material within the chest that appeared to fit the shape of a marker (Figures 3(a)-(b)). The patient was immediately taken to the operating room for direct laryngoscopy and neck exploration. Direct laryngoscopy revealed a two-centimeter

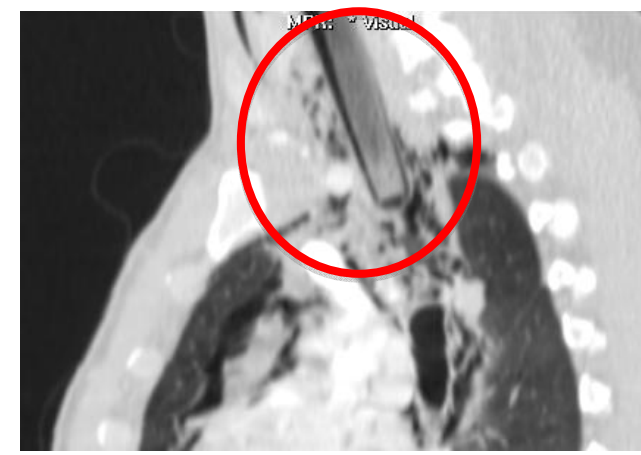

(a)

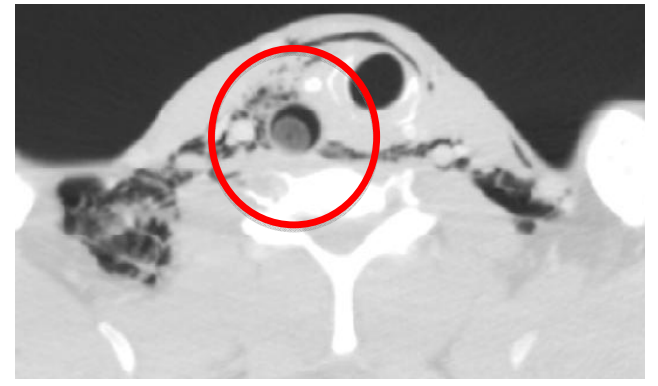

(b)

Figure 3. (a) \& (b): Non-contrast CT demonstrating foreign body extending into the thoracic cavity with air tracking to surrounding soft tissues and mediastinum, displacing airway to a left lateral position. Line encircles foreign body. 
pharyngotomy on the left lateral pharyngeal wall, extending from the level of the mid base of tongue toward the left arytenoid. An anterior commissure laryngoscope was directed toward the pharyngotomy, and a marker was clearly identified within this space extending toward the trachea. The marker was retrieved with laryngoscopic biopsy forceps and measured approximately twelve centimeters in length (Figure 4). Subsequent neck exploration was unrevealing. The pharyngotomy was left to close primarily with drains in place in the neck while the patient was kept with nothing per mouth.

The patient was placed on antibiotic prophylaxis against mediastinitis, although he remained stable and afebrile throughout his hospital course. Repeat CT scan of the neck and chest and a gastrograffin swallow study performed one week later showed no evidence of a pharyngomediastinal leak, and the patient's diet was slowly advanced. He was eventually discharged back to prison on postoperative day ten without any major sequelae from this incident. The patient later admitted to ingestion of a dry erase marker while under observation in the trauma bay.

\section{DISCUSSION}

Urgent diagnosis and treatment of pharyngeal injuries is essential for initiation of treatment as soon as possible to prevent such life-threatening complications of retropharyngeal abscess, mediastinitis, and/or airway compromise. Multiple studies of hypopharyngeal and cervical esophageal wounds have found that antibiotics within 12 - 24 hours from the time of injury are vital for the best possible outcome [1-3]. Pain is the most common presenting symptom of pharyngeal perforation, as was with our first case. There are no specific diagnostic signs for pharyngoesophageal injuries. Signs suggestive of injury include odynophagia, subcutaneous emphysema, and hematemesis or hemoptysis. Diagnosis can be difficult in patients that are obtunded, severely obese, psychotic, or are unstable. If there is any suspicion of hypopharyngeal or esophageal injury such as in penetrating injuries to the neck or torso, contrasted radiologic studies and endo-

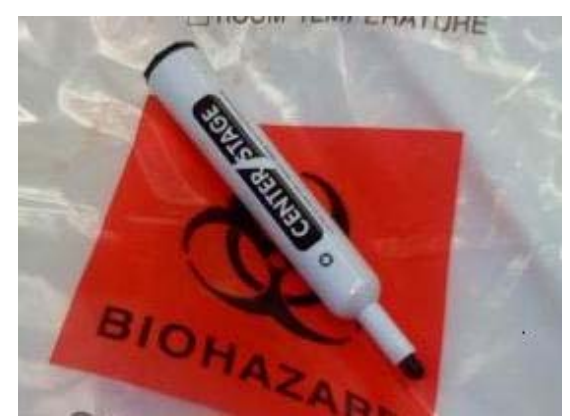

Figure 4. Marker retrieved from pharyngotomy during Direct Laryngoscopy. scopy are indicated. Fiberoptic endoscopic examination is the best screening tool for recognition of a possible hypopharyngeal mucosal violation, and is necessary if suspicion is high, as contrasted radiologic studies frequently miss hypopharyngeal injuries [4-6]. Endoscopic examination will also reveal the exact size and location of the injury, which is necessary to determine further workup and management.

Any trauma to the head and neck should include a thorough examination to assess each anatomic location and determine what anatomic planes and structures may have been violated. Injury to the airway or esophagus is among the most worrisome injuries, as esophageal injury has been associated with a high incidence of life-threatening mediastinitis [2,4,5,7]. Lacerations of the hypopharynx or cervical esophagus allow air, saliva, and food particles to enter into the fascial planes of the neck. Gravity and negative intrathoracic pressure facilitate the transmission of infected material into the deep neck spaces and mediastinum from the funnel formed by the hypopharynx and cervical esophagus.

Injury to the pharynx most commonly occurs after instrumentation or foreign body ingestion, with rare reports of spontaneous perforation following forceful vomiting or blunt trauma [1,7]. Pharyngeal injury has also been reported as a presenting sign of child abuse, and trauma to the intra-oral region from abuse has been shown to be highly associated with abuse-related death [8]. Traumatic pharyngeal injuries can occur as a result of penetrating or blunt trauma; however, blunt trauma has been shown to be a rare cause of pharyngoesophageal injury, accounting for less than $1 \%$ occurrence [2]. The outcome in penetrating trauma to the pharynx is typically more favorable, as blunt trauma often causes a larger disruption of the tissues, commonly with extent into the cervical esophagus [7]. Pharyngeal and cervical esophageal injuries have a much better prognosis than the thoracoabdominal esophagus.

Indication for primary closure of any pharyngeal injury depends on the location and extent, and is typically recommended for large tissue disruptions that are not well-approximated, or tears that extend into the esophagus. Niezgoda and collegues [9] reviewed 11 cases of pharyngoesophageal perforation following blunt neck trauma. They suggested non-operative management of antibiotics and fasting be reserved for patients with small perforations less than two centimeters and limited to the pharynx alone. Large perforations or perforations that involve the esophageal inlet or esophagus exclusively have been found to be best treated surgically [7,9]. Stanley et al. [3] in a study of 70 patients with pharyngeal-cervical esophageal injuries reported no difference in outcome between observation and surgery with an injury located in the hypopharynx above the arytenoid 
cartilage of the larynx. However, 22\% of patients with pharyngeal injuries below the level of the arytenoids and $39 \%$ of patients with cervical esophageal injuries developed deep infections or fistula. The authors concluded that injuries above the level of the arytenoid cartilage can be routinely managed nonoperatively, while all injuries below this level should be surgically repaired.

Both cases of pharyngeal trauma presented here were left to close primarily due to nature of the injuries. The lacerations were well-approximated, two centimeters or less, were above the level of the arytenoids, and did not involve the cervical esophagus. No significant sequelae occurred in either case, and both patients were tolerating oral intake without evidence of pharyngeal leak into surrounding tissues on clinical or radiologic examination. Although our first patient also had significant subcutaneous air within the soft tissues surrounding the pharynx, we decided on conservative management as surgical emphysema in the neck and surrounding the great vessels occurs without complication in post-operative patients following head and neck procedures such as neck dissection. The patient was observed for an extended period of time and developed no respiratory compromise or neurologic symptoms.

In conclusion, pharyngeal perforations are most commonly seen following penetrating trauma to the neck, but have also been reported in cases of blunt trauma to the neck, child abuse, and forceful vomiting. A high index of suspicion is warranted to pursue detection of injuries and prevention of devastating complications such as mediastinitis. Presenting symptoms include neck pain, odynophagia, hoarseness or airway distress often with subcutaneous emphysema found on physical exam. Treatment options must be individualized for each patient depending on extent of injury, and range from conservative therapy with analgesics, antibiotics, and fasting, to primary closure in the operating room. Conservative management is a safe and effective treatment for small pharyngeal lacerations not involving the cervical esophagus.

\section{REFERENCES}

[1] Roh, J.-L. and Park, C.I. (2008) Spontaneous pharyngeal perforation after forceful vomiting: The difference from classic Boerhaave's syndrome. Clinical and Experimental Otorhinolaryngology, 1, 174-176. doi:10.3342/ceo.2008.1.3.174

[2] Demetriades, D., Velmahos, G.G. and Asensio, J.A. (2001) Cervical pharyngoesophageal and laryngotracheal injuries. World Journal of Surgery, 25, 1044-1048. doi:10.1007/s00268-001-0057-9

[3] Stanley, R.B. Jr., Armstrong, W.B., Fetterman, B.L. and Shindo, M.L. (1997) Management of external penetrating injuries into the hypopharyngeal-cervical esophageal funnel. Journal of Trauma-Injury Infection \& Critical Care, 42, 675-679. doi:10.1097/00005373-199704000-00016

[4] Fetterman, B.L., Shindo, M.L., Stanley, R.B. Jr., Armstrong, W.B. and Rice, D.H. (1995) Management of traumatic hypopharyngeal injuries. Laryngoscope, 105, 8-13. doi:10.1288/00005537-199501000-00005

[5] Luqman, Z., Khan, M.A.M. and Nazir, Z. (2005) Penetrating pharyngeal injuries in children: Trivial trauma leading to devastating complications. Pediatric Surgery International, 21, 432-435. doi:10.1007/s00383-005-1447-0

[6] Ahmed, N., Massier, C., Tassie, J., Whalen, J. and Chung, R. (2009) Diagnosis of penetrating injuries of the pharynx and esophagus in the severely injured patient. Journal of Trauma-Injury Infection \& Critical Care, 67, 152-154. doi:10.1097/TA.0b013e31817e611d

[7] Hagr, A., Kamal, D. and Tabah, R. (2003) Pharyngeal perforation caused by blunt trauma to the neck. Canadian Journal of Surgery, 46, 57-58.

[8] Lin, H.W., Wieland, A.M. and Ostrower, S.T. (2009) Child abuse presenting as oral cavity bruising. Otolaryngology - Head Neck Surgery, 141, 290-291. doi:10.1016/j.otohns.2009.03.018

[9] Niezgoda, J.A., McMenamin, P. and Graeber, G.M. (1990) Pharyngoesophageal perforation after blunt neck trauma. The Annals of Thoracic Surgery, 50, 615-617. doi:10.1016/0003-4975(90)90199-G 